\title{
The S.T.O.N.E. Score: A new assessment tool to predict stone free rates in ureteroscopy from pre-operative radiological features
}

Wilson R. Molina, Fernando J. Kim, Joshua Spendlove, Alexandre S. Pompeo, Stefan Sillau, David E. Sehrt

Department of Urology (WRM, FJK, ASP), Denver Health Medical Center, Denver; Department of Urology (WRM, FJK, JS, DES) and Department of Statistics (SS), University of Colorado, Aurora, CO, USA and Department of Urology (ASP), ABC Medical School, Sao Paulo, Brazil

\section{ABSTRACT}

Objective: To develop a user friendly system (S.T.O.N.E. Score) to quantify and describe stone characteristics provided by computed axial tomography scan to predict ureteroscopy outcomes and to evaluate the characteristics that are thought to affect stone free rates.

Materials and Methods: The S.T.O.N.E. score consists of 5 stone characteristics: (S) ize, (T)opography (location of stone), (O)bstruction, (N)umber of stones present, and (E)valuation of Hounsfield Units. Each component is scored on a 1-3 point scale. The S.T.O.N.E. Score was applied to 200 rigid and flexible ureteroscopies performed at our institution. A logistic model was applied to evaluate our data for stone free rates (SFR). Results: SFR were found to be correlated to S.T.O.N.E. Score. As S.T.O.N.E. Score increased, the SFR decreased with a logical regression trend $(\mathrm{p}<0.001)$. The logistic model found was $S F R=1 /\left(1+e^{\wedge}(-z)\right)$, where $z=7.02-0.57^{*}$ Score with an area under the curve of 0.764. A S.T.O.N.E. Score $\leq 9$ points obtains stone free rates $>90 \%$ and typically falls off by $10 \%$ per point thereafter.

Conclusions: The S.T.O.N.E. Score is a novel assessment tool to predict SFR in patients who require URS for the surgical therapy of ureteral and renal stone disease. The features of S.T.O.N.E. are relevant in predicting SFR with URS. Size, location, and degree of hydronephrosis were statistically significant factors in multivariate analysis. The S.T.O.N.E. Score establishes the framework for future analysis of the treatment of urolithiasis.

\section{ARTICLE INFO}

Key words:

Ureteroscopy; Urolithiasis; Lithotripsy, Laser; Logistic Models; Nomograms

Int Braz J Urol. 2014; 40: 23-9

Submitted for publication:

March 25, 2013

Accepted after revision:

September 09, 2013

\section{INTRODUCTION}

The prevalence of stone disease is increasing not only in the United States, but worldwide $(1,2)$. The total cost of treating stones in the US currently exceeds five billion dollars annually (2). With this increase in incidence and the cost of healthcare continuing to rise, new approaches to stone disease may be necessary.
Extra-corporeal shockwave lithotripsy (ESWL) is the present mainstay of treatment for intra-renal and proximal ureteral stones $\leq 1 \mathrm{~cm}$ in diameter due to its relatively low complication rate and high success rate $(3,4)$. Current guidelines from American Association of Urology (AUA) recommend ESWL as the first treatment option for proximal ureteral calculi. Modern stone therapy should ensure high effectiveness combined with 
low complication rate. URS has become common in the treatment of proximal and intrarenal stones $<20 \mathrm{~mm}$ as a result of technological improvements in visualization and laser technology $(5,6)$.

Multiple studies have examined the predictive factors associated with ESWL outcomes as well as nomograms to predict stone free rates (SFR) $(7,8)$. To our knowledge there is a lack of user friendly assessment tools to determine the complexity of ureteral and kidney stones and determine SFR after URS. Factors that influence SFR are pivotal to elect best treatment modality for stone disease. We propose a simple method to estimate the SFR for URS to treat urolithiasis.

\section{MATERIAL AND METHODS}

A retrospective review of patients who underwent URS was approved by the institutional review board. Known factors associated with stone free rates were used to establish a simplified method to estimate SFR. Inclusion criteria consisted of consecutive patients with ureteral and renal stones with preoperative non-contrast computed axial tomography (CT-KUB). Patients with anatomical abnormalities such as duplicated ureters, horseshoe kidney, ureteral strictures were excluded from analysis.

Stone free was defined as absent of stone fragments or fragments $\leq 2 \mathrm{~mm}$ post URS after rigorous endoscopic inspection and real time fluoroscopy with the capability of high magnification imaging (9). If combined endoscopic visualization and fluoroscopy was sub-optimal, a CT scan was obtained to confirm stone free status. If a patient was considered stone-free intraoperatively and did not have a postoperative CT scan, a chart reviewed was performed. These patients were considered clinically stone-free if no admissions or visits to the emergency were found during follow-up.

The S.T.O.N.E. Score is a proposed system to predict the stone free status of a patient from preoperative characteristics available on CT-KUB: (S)ize of the stone, (T)opography or location, degree of (0)bstruction of the urinary system, (N) umber of stones, and (E)valuation of Hounsfield units. Higher scores indicate higher complexity and assumingly lower stone free rates. Each feature from the CT was graded on a 1-3 point scale as described in Table-1. In cases with multiple calculi, the stone with the highest grade for each feature was recorded. All scores were assigned after a consensus of two observers.

Stone (S)ize was the initial variable in S.T.O.N.E. Score. Size was measured as the maximum diameter of the stone in any plane. Our scoring system was based on the AUA's stratification to estimate ureteral stone passage by size (3). One point was given for stones $<5 \mathrm{~mm}, 2$ points were given to stones $\geq 5 \mathrm{~mm}$ and $<10 \mathrm{~mm}$, and 3 points were given to stones $\geq 10 \mathrm{~mm}$.

(T)opography or location was another factor included that affects stone free rates in URS. While distal stones tend to be easily treated with SFR of 90\%, stones in the proximal ureter and within the kidney may be challenging for URS (4).

Table 1 - S.T.O.N.E. Score.

\begin{tabular}{|c|c|c|c|}
\hline Feature & $1 \mathrm{pt}$. & $2 \mathrm{pt}$. & 3 pt. \\
\hline (S)ize & $<5 \mathrm{~mm}$ & $5-10 \mathrm{~mm}$ & $>10 \mathrm{~mm}$ \\
\hline (T)opography & Distal to Mid-Ureter & $\begin{array}{l}\text { Proximal Ureter through Mid and } \\
\text { Upper Pole }\end{array}$ & Lower Pole \\
\hline (0)bstruction & $\begin{array}{l}\text { Preoperative Stent or No } \\
\text { Hydronephrosis }\end{array}$ & Grade 1-2 & Grade 3-4 \\
\hline (N)umber of stones & 1 stone & 2 stones & $\geq 3$ stones \\
\hline (E)valuation of $\mathrm{HU}$ & $<750 \mathrm{HU}$ & $750-1000 \mathrm{HU}$ & $>1000 \mathrm{HU}$ \\
\hline
\end{tabular}


Mid and upper pole renal stones were considered less challenging than the lower pole stones, since several factors may challenge the surgeon i.e. infundibular angle and diameter. Therefore, we scored 1 point for distal and mid ureter stones, 2 points for proximal ureter, mid pole, and upper pole stones, and 3 points to lower pole stones.

(0)bstruction was scored as the degree of hydronephrosis in the collecting system and the presence of a stent. The greater the degree of hydronephrosis, the higher is the obstruction and the lower the SFR after URS. We implemented a modified version of the Society for Fetal Urology Hydronephrosis Grading System (10). Grade 1 hydronephrosis was defined as local dilation of the ureter. Grade 2 included ureteral and renal pelvis dilatation. Grade 3 also included calix dilatation. Grade 4 exhibited parenchymal thinning. The benefits of pre-stenting prior to ureteroscopy have been previously shown (11). The reasons for pre stenting in our population of patients included: severe preoperative pain, infection managed and treated prior ureteroscopy, and from outside referrals to our safety net hospital. All patients had their stents for at least 2 weeks prior to ureteroscopy. Patients with pre-stenting or no hydronephrosis received 1 point, Grade 1-2 hydronephrosis received 2 points, and Grade 3-4 received 3 points.

The (N)umber of stones is well known to influence treatment. Stones greater than $2 \mathrm{~mm}$ were counted into the score. Patients with 1 stone were assigned 1 point, patients with 2 stones were assigned 2 points, and patients with $\geq 3$ stones were assigned 3 points.

Finally, (E)valuation of the Hounsfield Units (HU) was calculated as the average Hounsfield Units instead of standard deviation or maximum attenuation (12). Hounsfield Units $<750$ received 1 point, between 750 and 1000 HU received 2 points, and 3 points for $\geq 1000 \mathrm{HU}$. These minimum and maximum values were chosen due to known SFR correlations $(13,14)$. Ureteroscopy and stone treatment was performed with the patient in the lithotomy position. A 7.5 Fr semirigid ureteroscope (Olympus Corporation, Tokyo, Japan) was used for stones in the distal and mid ureter. For proximal ureteral and renal stones, an access sheath (Cook Medical, Bloomington, IN) and a 9 Fr flexible (Olympus Corporation, Tokyo, Japan) ureteroscope was used. Prior to 2009 a fiber optic flexible ureteroscopy (Karl Storz Flex$-\mathrm{X}^{\mathrm{TM}}$, Inc., Tuttlingen, Germany) was utilized. The stone was assessed and laser lithotripsy was performed when necessary using a Holmium Laser (Donnier Medilas, Kennesaw, GA) set at 6-10W with a 270 or $400 \mu \mathrm{m}$ laser fiber (Gyrus ACMI, Southborough, MA). Stone fragments were then removed by a stone retrieval basket (Boston Scientific, Natick, MA). Following complete stone removal, stone free status was evaluated with the methods previously described.

Statistical analyses used to construct the S.T.O.N.E. Score were performed using the R version 2.11 software (the R foundation for Statistical Computing, Vienna, Austria). The goodness of fit using the Area Under the Curve (AUC) for the S.T.O.N.E. Score was performed using the ROCR statistical package (15). Data are presented as average \pm standard deviation or frequency (percentage of total). A p-value $<0.050$ was considered significant. The corresponding author had full access to all the data in the study and takes responsibility for the integrity of the data and the accuracy of the data analysis.

\section{RESULTS}

A total of 200 URS procedures from August 2006 to January 2012 were assessed by the S.T.O.N.E. Score. Patients age was $44.1 \pm 13.9$, were equally represented by gender (male:female of $87: 113)$ and obesity $\left(\mathrm{BMI}>30 \mathrm{~kg} / \mathrm{m}^{2}\right)$. Often, cases were unilateral stones, were not favored to either side of the urinary tract (right:left:bilateral of 106:91:3), and had a mean stone size of $9.3 \pm 5.9$.

The overall SFR in the entire cohort was $82 \%$. A total of 28 patients were diagnosed with residual stones intra-operatively and another 8 patients were found to have residual stones on postoperative CT. Postoperative imaging (CT) demonstrated 67 patients were stone free. The remaining 97 patients were considered stone free following ureteroscopy and did not seek further treatment in Urology or the Emergency Department after a mean follow-up time of 20 months. 
The multivariate regression demonstrated that as stones became larger, more proximal to the lower pole, caused greater hydronephrosis, increased in number, and had higher Hounsfield units, the probability of becoming stone free decreased. According to the multivariate model, stone size, location, and hydronephrosis grades had similar impact (weighting) on the stone free status (Table-2). Stone size and location were significant factors affecting stone free rates $(p<0.05)$. The severity of hydronephrosis was nearly significant $(p=0.07)$. The accuracy of the multivariate model by the area under the curve was 0.837 .

The S.T.O.N.E. Score was established as a simplified assessment tool to predict stone free rates (Table-3). The formula derived from the logistic model for stone free rate was as follows: SFR $=1 /(1+\exp (-z))$, where $z=7.02-0.57^{*}$ Score. As the S.T.O.N.E. Score increases, the stone free rate decreases $(p<0.001)$ (ESM 1). The accuracy of the S.T.O.N.E. Score was comparable to the multivariate model $($ AUC $=0.764)$ (Figure-1).

Table 2 - Multivariate Logistic Regression Model.

\begin{tabular}{lccc}
\hline Variable & Estimate & $95 \% \mathrm{Cl}$ & P-value \\
\hline $\mathrm{S}$ & 1.1 & $-1.8-0.4$ & 0.002 \\
$\mathrm{~T}$ & 1.3 & $-2.1-0.6$ & $<0.001$ \\
0 & 0.5 & $-1.1-0.1$ & 0.077 \\
$\mathrm{~N}$ & 0.2 & $-0.7-0.3$ & 0.472 \\
$\mathrm{E}$ & 0.0 & $-0.5-0.5$ & 0.926 \\
\hline
\end{tabular}

$\mathrm{AUC}=0.806$

Table 3 - S.T.O.N.E. score compared to URS outcomes.

\begin{tabular}{lcccc}
\hline S.T.O.N.E. Score & N & 200 Patients & S.T.0.N.E. Score & Rounded Score \\
\hline 5 & 8 & $100 \%$ & $99 \%$ & $100 \%$ \\
6 & 18 & $100 \%$ & $97 \%$ & $100 \%$ \\
7 & 30 & $100 \%$ & $95 \%$ & $100 \%$ \\
8 & 30 & $93 \%$ & $92 \%$ & $90 \%$ \\
9 & 27 & $81 \%$ & $87 \%$ & $90 \%$ \\
10 & 25 & $64 \%$ & $78 \%$ & $80 \%$ \\
11 & 34 & $74 \%$ & $67 \%$ & $70 \%$ \\
12 & 19 & $68 \%$ & $54 \%$ & $50 \%$ \\
13 & 6 & $67 \%$ & $40 \%$ & $40 \%$ \\
14 & $33 \%$ & $28 \%$ & $30 \%$ \\
15 & 3 & - & $18 \%$ & $20 \%$ \\
\hline
\end{tabular}


Figure 1 - Receiver operator curves for the S.T.O.N.E. Score (AUC $=0.764$ ) and the Multivariate Regression Model (AUC $=0.806$ ).

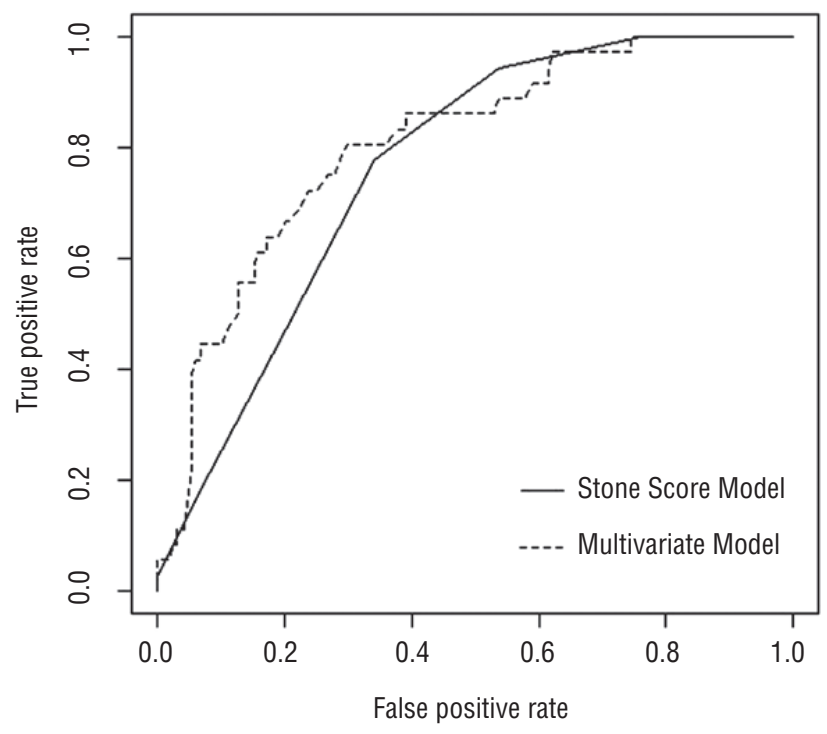

DISCUSSION

The development of statistical models provides physicians with new insight into patient planning and counseling. Furthermore, models typically standardize terminology and improve communication in reports. In stone disease, features affecting the success of ESWL such as skin-to-stone distance, optimal location, density (Hounsfield Units), and size have all been adequately reported $(7,14,16)$. On the other hand, there is presently a paucity of nomograms or score systems to predict stone free rates for URS.

The S.T.O.N.E. Score is a user friendly model to predict SFR post URS with laser lithotripsy. It further establishes a standardized terminology for reporting urolithiasis characteristics. We attempt to identify the five most important preoperative features that could be related to surgical outcomes in URS: (S)ize, (T)opography/location, (0) bstruction, (N)umber of stones, and (E)valuation of Hounsfield Units $(12,17)$.

The S.T.O.N.E. Score remains consistent with $77 \%$ of the present literature as stone size is usually described by the maximum diameter (18). A recent study revealed stones $>20$ and $<40 \mathrm{~mm}$ treated by URS reported a stone free rate of 100\% with mean 1.4 number of procedures (19). The general consensus in the literature cites a negative correlation between stone size and SFR and is included in the S.T.O.N.E. Score $(5,6)$.

Stone location is an important factor in the success of URS, especially lower pole stones $(5,6,18)$. Although visualization of these stones may be possible with flexible endoscopes, the acute pelvic infundibular angle may prevent access to the stone, especially with less deflection of the ureteroscope when the basket or laser fiber is inside of the working channel. Generally, SFR for lower pole stones have typically been reported around 80\% $(5,6,18)$. Other intra-renal and proximal ureteral stones have been reported with a stone free rate of approximately 90\% (18). More distal stones from the mid ureter to the ureterovesical junction stones have been reported with a stone free rate $>95 \%$ (18). These stones have been easily managed with rigid ureteroscopy. This data along with our own observations have led to the stratification levels established for the S.T.O.N.E. Score.

Impacted stones and hydronephrosis are other mechanisms that diminish stone clearance. Although impacted stones are difficult to be evaluated on CT, the presence and degree of hydronephrosis may be an indirect indicator. Additionally, prolonged obstruction increases the amplitude and frequency of ureteral peristalsis contractions resulting in histological changes including smooth muscle hypertrophy and collagen deposition (20). These histological changes may affect ureteroscopy instrumentation limiting the ability to manipulate stones proximal at the level of obstruction. Stenting has shown to improve SFR compared to non-stented patients (12). Rubenstein et al. saw a stone free rate of 78 and 54\% for patients with and without pre-stenting respectively. We have accounted for the pre-stenting effect by incorporating this into the (0)bstruction score. The (0)bstruction score was further graded by a modified Society for Fetal Urology score in order to quantify the severity of hydronephrosis. There are few grading systems objectively quantifying hydronephrosis. Our system is simple to implement and correlates with stone free rates. Although there has been little data investigating the effect of hydronephrosis 
in URS, our data shows this is a significant factor and is included in the S.T.O.N.E. Score.

The number of stones has been shown to be significant in other studies (21). A number of methods have been developed to describe stone burden by incorporating both the size and number of stones $(21,22)$. The description of total stone burden applies two dimensional measurements using rectangular and elliptical approximations (18). Furthermore, a three dimension description of stone burden would be the most accurate system, but the difficulty in implementation and software availability has inhibited its use at the present time. For simplicity, we chose to incorporate the number of stones to describe stone burden in our nomogram.

Stone hardness and Hounsfield Units on CT have often been overlooked in URS. Hounsfield Units has commonly been reported to be a significant factor in shock-wave lithotripsy (23). Chung et al. reported a statistical difference between successful and unsuccessful ESWL (675.29 versus 1075.00, respectively). This trend has been observed in URS but without statistical significance. A study found that stones successfully treated by URS had a mean of $858 \mathrm{HU}$ while stones that were unsuccessfully treated had a mean of 1115 HU (6). Stone composition increasing density and hardness may prolong OR time.

The limitations of this study include a retrospective single institution analysis. Furthermore, a standardized definition and methodology of evaluating stone free status is lacking currently in the literature. The clinical significance of residual stone size is presently unknown with stone free definitions most commonly ranging from the complete absent of stones to residual fragments $<4 \mathrm{~mm}$ (24). Our definition of stone free corroborates with a study that has shown that stones $>$ $2 \mathrm{~mm}$ are related to a recurrent stone event (25). A number of radiological methods for evaluating stone free status include CT, KUB (fluoroscopy), and Ultrasound (24). Although CT may be the best imaging modality to evaluate presence and or burden of stone post treatment, certainly, it is not free of limitations. Furthermore, the presence of ureteral stents may obscure the presence of small stone fragments following URS (26). This pragma- tic method of limiting postoperative imaging to questionable and complicated cases is commonly accepted and minimizes radiation and costs to the patient $(9,27)$. Aggressive endoscopic inspection combined with high magnification fluoroscopy has shown high sensitivity and specificity in evaluating $0-4 \mathrm{~mm}$ stones $(9,27)$. Nonetheless, the S.T.O.N.E. Score correlates with SFR and can be applied in daily practice without complex instrumentation or time consuming calculations. Future work is needed to validate the S.T.O.N.E. Score.

\section{CONCLUSIONS}

The S.T.O.N.E. Score is a novel assessment tool to predict SFR in patients undergoing URS. Features of S.T.O.N.E. (stone size, location, and degree of hydronephrosis) were relevant in predicting SFR with URS. The S.T.O.N.E. Score establishes the framework for future analysis for the treatment of urolithiasis.

\section{ABBREVIATIONS}

AUC $=$ Area Under the Curve

ESWL $=$ Extracorporeal shockwave lithotripsy

$\mathrm{SFR}=$ Stone Free Rate

URS = Ureteroscopy

\section{CONFLICT OF INTEREST}

Wilson Molina - Boston Scientific Course Proctor and Supported Fellowship

Fernando J. Kim - Olympus Proctor

Joshua Spendlove, Alexandre Pompeo, Stefan Sillau and David Sehrt - None declared.

\section{REFERENCES}

1. Nowfar S, Palazzi-Churas K, Chang DC, Sur RL: The relationship of obesity and gender prevalence changes in United States inpatient nephrolithiasis. Urology. 2011; 78: 1029-33.

2. Turney BW, Reynard JM, Noble JG, Keoghane SR: Trends in urological stone disease. BJU Int. 2012; 109: 1082-7.

3. Preminger GM, Tiselius HG, Assimos DG, Alken P, Buck C, Gallucci M, et al.: 2007 guideline for the management of ureteral calculi. J Urol. 2007; 178: 2418-34. 
4. Tiselius HG, Ackermann D, Alken P, Buck C, Conort P, Gallucci M; et al.: Guidelines on urolithiasis. Eur Urol. 2001; 40: 362-71.

5. Bagley DH: Expanding role of ureteroscopy and laser lithotripsy for treatment of proximal ureteral and intrarenal calculi. Curr Opin Urol. 2002; 12: 277-80.

6. Hussain M, Acher P, Penev B, Cynk M: Redefining the limits of flexible ureterorenoscopy. J Endourol. 2011; 25: 45-9.

7. Kim FJ, Rice KR: Prediction of shockwave failure in patients with urinary tract stones. Curr Opin Urol. 2006; 16: 88-92.

8. Wang M, Shi Q, Wang X, Yang K, Yang R: Prediction of outcome of extracorporeal shock wave lithotripsy in the management of ureteric calculi. Urol Res. 2011; 39: 51-7.

9. Schoenthaler M, Wilhelm K, Katzenwadel A, Ardelt P, Wetterauer $\mathrm{U}$, Traxer 0, et al.: Retrograde intrarenal surgery in treatment of nephrolithiasis: is a $100 \%$ stone-free rate achievable? J Endourol. 2012; 26: 489-93.

10. Fernbach SK, Maizels M, Conway JJ: Ultrasound grading of hydronephrosis: introduction to the system used by the Society for Fetal Urology. Pediatr Radiol. 1993; 23: 478-80.

11. Rubenstein RA, Zhao LC, Loeb S, Shore DM, Nadler RB: Prestenting improves ureteroscopic stone-free rates. J Endourol. 2007; 21: 1277-80.

12. Kacker R, Zhao L, Macejko A, Thaxton CS, Stern J, Liu $\mathrm{JJ}$, Nadler RB: Radiographic parameters on noncontrast computerized tomography predictive of shock wave lithotripsy success. J Urol. 2008; 179: 1866-71.

13. Gupta NP, Ansari MS, Kesarvani P, Kapoor A, Mukhopadhyay $S$ : Role of computed tomography with no contrast medium enhancement in predicting the outcome of extracorporeal shock wave lithotripsy for urinary calculi. BJU Int. 2005; 95: 1285-8.

14. Wen CC, Nakada SY: Treatment selection and outcomes: renal calculi. Urol Clin North Am. 2007; 34: 409-19.

15. Sing T, Sander 0 , Beerenwinkel N, Lengauer T: ROCR: visualizing classifier performance in R. Bioinformatics. 2005; 21: 3940-1.

16. Joseph P, Mandal AK, Singh SK, Mandal P, Sankhwar SN, Sharma SK: Computerized tomography attenuation value of renal calculus: can it predict successful fragmentation of the calculus byextracorporeal shock wave lithotripsy? A preliminary study. J Urol. 2002; 167: 1968-71.

17. Macejko A, Okotie OT, Zhao LC, Liu J, Perry K, Nadler RB: Computed tomography-determined stone-free rates for ureteroscopy of upper-tract stones. J Endourol. 2009; 23: 379-82.
18. Patel SR, Nakada SY: Quantification of preoperative stone burden for ureteroscopy and shock wave lithotripsy: current state and futurerecommendations. Urology. 2011; 78: 282-5.

19. Takazawa R, Kitayama S, Tsujii T: Successful outcome of flexible ureteroscopy with holmium laser lithotripsy for renal stones $2 \mathrm{~cm}$ or greater. Int J Urol. 2012; 19: 264-7.

20. Kinn AC, Lykkeskov-Andersen $\mathrm{H}$ : Impact on ureteral peristalsis in a stented ureter. An experimental study in the pig. Urol Res. 2002; 30: 213-8.

21. Herrera-Gonzalez G, Netsch C, Oberhagemann K, Bach T, Gross AJ: Effectiveness of single flexible ureteroscopy for multiple renal calculi. J Endourol. 2011; 25: 431-5.

22. Breda A, Ogunyemi O, Leppert JT, Schulam PG: Flexible ureteroscopy and laser lithotripsy for multiple unilateral intrarenal stones. Eur Urol. 2009; 55: 1190-6.

23. Park YI, Yu JH, Sung LH, Noh CH, Chung JY: Evaluation of possible predictive variables for the outcome of shock wave lithotripsy of renal stones. Korean J Urol. 2010; 51: 713-8.

24. Deters LA, Jumper CM, Steinberg PL, Pais VM Jr.: Evaluating the definition of "stone free status" in contemporary urologic literature. Clin Nephrol. 2011; 76: 354-7.

25. Raman JD, Bagrodia A, Gupta A, Bensalah K, Cadeddu JA, Lotan Y, et al.: Natural history of residual fragments following percutaneous nephrostolithotomy. J Urol. 2009; 181: 1163-8.

26. Tang VC, Attwell-Heap A: Computed tomography versus ureteroscopy in identification of renal tract stone with ureteral stent in situ. Ann R Coll Surg Engl. 2011; 93: 639-41.

27. Portis AJ, Laliberte MA, Drake S, Holtz C, Rosenberg MS, Bretzke CA: Intraoperative fragment detection during percutaneous nephrolithotomy: evaluation of high magnification rotationalfluoroscopy combined with aggressive nephroscopy. J Urol. 2006; 175: 162-5; discussion 165-6. Erratum in: J Urol. 2006; 175(3 Pt 1): 1176.

Correspondence address:

Wilson Molina, MD

Chief of Endourology,

Urology Department, Denver Health Hospital 777 Bannock St, Denver, C0 80204, USA

Fax: + 1303 436-6572

E-mail: wilson.molina@dhha.org 